\title{
Metabolic labeling of enterovirus 71 with quantum dots for the study of virus receptor usage
}

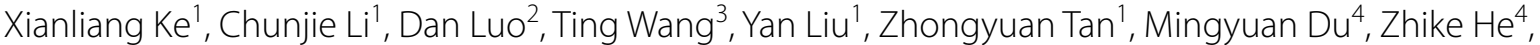 \\ Hanzhong Wang ${ }^{1}$, Zhenhua Zheng ${ }^{1 *}$ and Yuan Zhang ${ }^{1 *}$
}

\begin{abstract}
Fluorescent labeling and dynamic tracking is a powerful tool for exploring virus infection mechanisms. However, for small-sized viruses, virus tracking studies are usually hindered by a lack of appropriate labeling methods that do not dampen virus yield or infectivity. Here, we report a universal strategy for labeling viruses with chemical dyes and Quantum dots (QDs). Enterovirus 71 (EV71) was produced in a cell line that stably expresses a mutant methionyl-tRNA synthetase (MetRS), which can charge azidonorleucine (ANL) to the methionine sites of viral proteins during translation. Then, the ANL-containing virus was easily labeled with DBCO-AF647 and DBCO-QDs. The labeled virus shows sufficient yield and no obvious decrease in infectivity and can be used for imaging the virus entry process. Using the labeled EV71, different functions of scavenger receptor class B, member 2 (SCARB2), and heparan sulfate (HS) in EV71 infection were comparatively studied. The cell entry process of a strong HS-binding EV71 strain was investigated by real-time dynamic visualization of EV71-QDs in living cells. Taken together, our study described a universal biocompatible virus labeling method, visualized the dynamic viral entry process, and reported details of the receptor usage of EV71.
\end{abstract}

Keywords: EV71, Quantum dots, Nanobiotechnology, Metabolic labeling, ANL, Heparan sulfate, SCARB2

\section{Introduction}

Human enterovirus 71 (EV71) is the major causative pathogen of the human hand, foot, and mouth disease (HFMD) [1]. EV71 infects millions of children in the Asian-Pacific area and causes thousands of deaths every year. Because HFMD often leads to severe neurological complications, such as brainstem encephalitis, meningitis, and poliomyelitis-like paralysis, EV71 is recognized as one of the most important neurotropic picornaviruses [1].

\footnotetext{
*Correspondence: zhengzh@wh.iov.cn; zhangyuan@wh.iov.cn ${ }^{1}$ CAS Key Laboratory of Special Pathogens and Biosafety, Center for Biosafety Mega-Science, Wuhan Institute of Virology, Chinese Academy of Sciences, Wuhan 430071, China

Full list of author information is available at the end of the article
}

Currently, no effective drugs have been developed for the EV71 virus. The cell entry process is a key step for viruses to establish successful infection in cells and usually provides promising targets for antiviral therapeutic intervention [2]. In recent decades, many cellular molecules, such as vimentin, SCARB2, PSGL-1, nucleolin, fibronectin, and heparan sulfate proteoglycans, have been proven to serve as cellular receptors for EV71 infection [3-7]. However, the mechanism of cell entry mediated by the receptors, as well as viral preferences among them, are not fully understood.

Virus tracking in living cells provides powerful tools for the investigation of the cell entry mechanism of many viruses [8]. The most challenging step of virus tracking is labeling viral particles without affecting their replication and infectivity. By genetic fusion of fluorescent proteins original author(s) and the source, provide a link to the Creative Commons licence, and indicate if changes were made. The images or other third party material in this article are included in the article's Creative Commons licence, unless indicated otherwise in a credit line to the material. If material is not included in the article's Creative Commons licence and your intended use is not permitted by statutory regulation or exceeds the permitted use, you will need to obtain permission directly from the copyright holder. To view a copy of this licence, visit http://creativecommons.org/licenses/by/4.0/. The Creative Commons Public Domain Dedication waiver (http://creativeco mmons.org/publicdomain/zero/1.0/) applies to the data made available in this article, unless otherwise stated in a credit line to the data. 
(FPs) or conjugation of fluorescent probes to viral structural proteins, many viruses have been successfully labeled [9]. However, small-sized viruses such as EV71 have benefited little from this technique due to their virological characteristics. For example, the relatively small size of the EV71 particles $(\sim 28 \mathrm{~nm})$ [1] and its structural proteins make FP-fusion an impossible mission. The molecular weight of the commonly used FPs $(\sim 27 \mathrm{kDa})$ is equal to or even exceeding that of the targeted viral proteins. So, fusion with FPs may easily prevent viral capsid protein monomers from multimerization into highly ordered viral particles. Consequently, this labeling strategy would be lethal for some small-sized viruses including EV71, thus, limited its application. As for chemical labeling, the target charged residues on viral particles are often essential for the receptor binding of EV71 [10, 11]. Several positively charged residues were reported to be responsible for virus binding to HS and PSGL-1 and even for virulence in patients and animal models [11-13]. Moreover, chemical labeling may interfere with the interaction between viruses and cells. To date, only a few studies focused on dynamic visualization of the EV71 infection process have been reported $[14,15]$.

Metabolic incorporation of unnatural amino acids (UAAs) is a novel strategy that provides opportunities to label viruses with minimal effects on virus infectivity. The methionine (Met) analog azidohomoalanine (AHA) is the most commonly used UAA for virus labeling. AHA can bind to the endogenous methionyl-tRNA synthetase (MetRS) instead of Met [16], thus, can be incorporated into viruses during viral protein translation. The progeny virus can be labeled with fluorescent probes through reaction with the azido group [14, 17-19]. However, the affinity of AHA to MetRS is 390-500-fold lower than that of Met. To eliminate the low labeling efficiency caused by the competition between AHA and Met, AHA-base virus labeling must be performed in Met-free medium [17, 19], which induces substantial protein abundance differences [20] and may affect cell growth or even virus yield significantly [21]. UAAs can also be incorporated into virus proteins through genetic code expansion in mammalian cells. However, the labeling system requires not only engineered aaRS/tRNA expression cassettes but also eRF1 mutants to improve the incorporation efficiency [22], making the whole system rather complicated.

Long-term dynamic tracking of the labeled virus is often limited by the photobleaching of traditional chemical dyes or FPs. Quantum dot (QD), an inorganic nanoparticle, is an alternative fluorescent material suitable for long-term imaging owing to its remarkable brightness and photostability. It has been widely used for the dynamic tracking of many viruses including retrovirus [23] and influenza virus [24].
In this study, we developed a novel virus labeling method and accomplished long-time dynamic visualization of the cell entry of EV71 through QD-labeling (Scheme 1). The labeling technique relies on a mutant MetRS and azidonorleucine (ANL) [25], another Met surrogate, to label viral particles during virus production. MetRS with an amino-acid binding site mutation (MetRS L274G) enables the Met tRNA to be charged with ANL instead of Met efficiently without the requirement of Met deletion [25]. When cells that express MetRS L274G were applied for virus production, cellular proteins, as well as the progeny viral proteins, could be modified by incorporating ANL. Then ANL-carrying viruses can be readily labeled with dibenzocyclooctynol (DBCO) probes including DBCO-QDs through click chemistry under physiological conditions with high efficiency [26]. Using the labeled viruses, the functions of two EV71 receptors during infection, SCARB2, and heparan sulfate (HS) were comparatively studied. Entry of a strong-HS-binding EV71 strain into SCARB2-knockout cells was also studied by long-term dynamic visualization.

\section{Methods \\ Cell lines and viruses}

RD (human rhabdomyosarcoma cells) and HeLa cell lines were maintained in Dulbecco's modified Eagle's medium (DMEM) supplemented with 10\% fetal bovine serum (FBS). Vero (African green monkey kidney cells) cell line was maintained in minimum essential medium (MEM) supplemented with 10\% FBS. Two SCARB2-knockout mouse fibroblast cell lines, namely, the L929-SCARB2KO and L929-EXT1KO cell lines, which were heparan sulfate deficient, were constructed as described previously [27]. L929, L929-SCARB2KO, and L929-EXT1KO cells were maintained in Dulbecco's modified Eagle's medium (DMEM) supplemented with $10 \%$ FBS. EV71 virus strain EV71 GZCII-P30 [27] was propagated in Vero or RD cells. After titering in RD cells, virus stocks were stored at $-80{ }^{\circ} \mathrm{C}$ until use.

\section{RD-MetRS* cell line construction}

All molecular cloning work was performed in E. coli strain DH5 $\alpha$. Briefly, the human methionyl-tRNA synthetase (MetRS) coding region was amplified from the cDNA of HEK-293T cells using primer MetRS-F and MetRS-R. Then, a leucine to glycine mutation at amino acid 272 was introduced into MetRS by overlappingPCR. Two DNA fragments were amplified using primer MetRS*-F(EcoRI) + MetRS*-2R or MetRS*-2 F + MetRS*$\mathrm{R}$ (NotI) respectively, and then fused together by PCR using primers MetRS*-F(EcoRI) + MetRS*-R(NotI). The MetRS mutant was cloned into the PiggyBac transposon vector (PB513B-1, System Biosciences, USA) between 


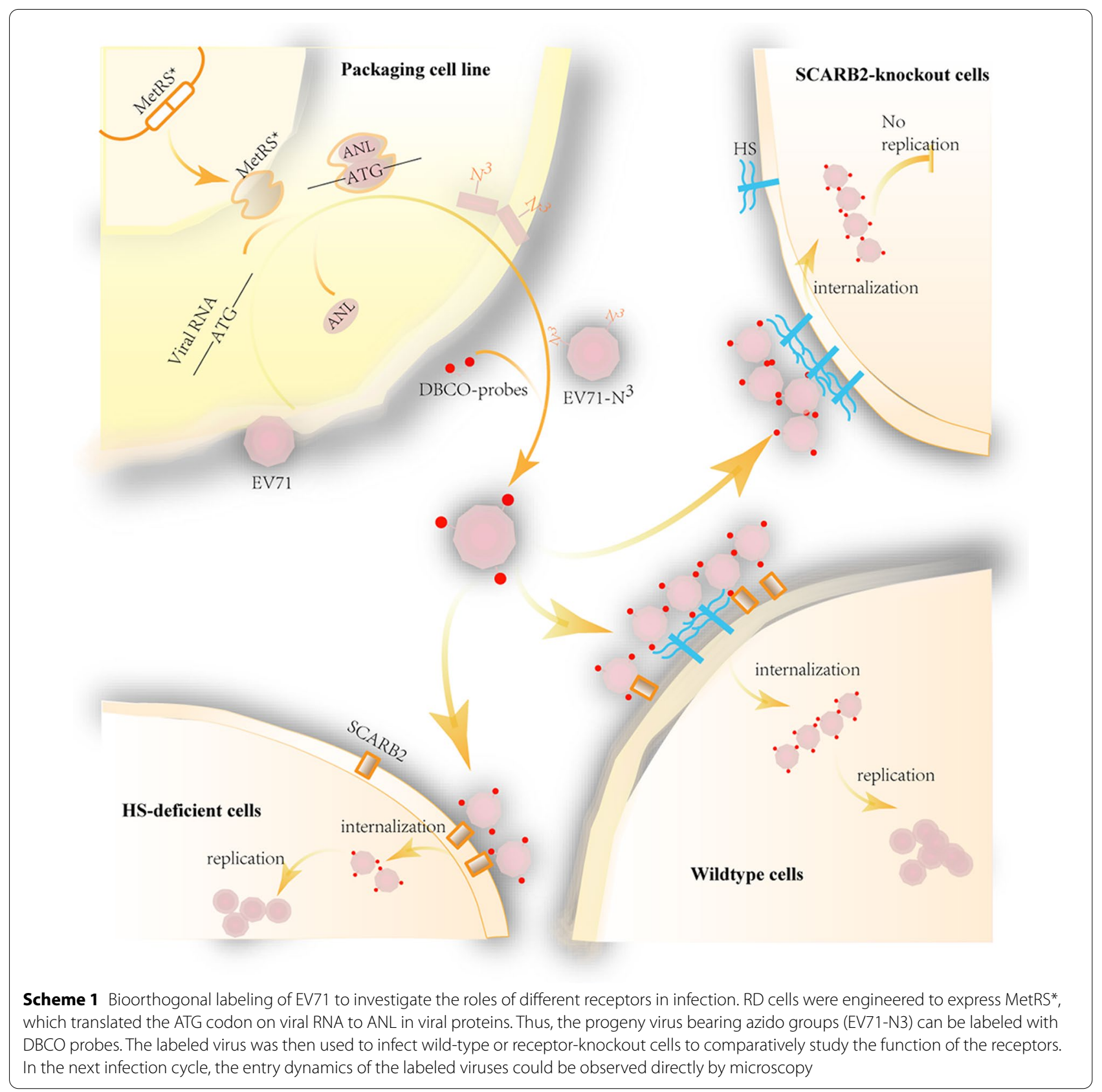

enzyme sites EcoRI and NotI through restriction cloning to generate PB-MetRS*. Primer sequences are listed in Additional file 1: Table S1.

The plasmid PB-MetRS* was transfected into the RD cell line along with Super PiggyBac Transposase (PB200PA-1, System Biosciences, USA) expression plasmid at a ratio of $2: 1$. The transposase will move the transposon which harbors the MetRS* and Pac (puromycin resistance gene) gene to the cell genome for stable expression. Transfected cells were screened with $4 \mu \mathrm{g} / \mathrm{mL}$ puromycin (Sangon Biotech, China) for 2 weeks to select MetRS"-expressing cells, which were designated RDMetRS* cells and used for virus labeling.

\section{Virus labeling and purification}

RD-MetRS* cells were cultured in normal medium for $24 \mathrm{~h}$. Then, EV71 was added at a multiplicity of 0.01 for infection at $37^{\circ} \mathrm{C}$ for $1 \mathrm{~h}$. After incubation, cells were cultured with normal medium supplemented with $1000 \mu \mathrm{M}$ ANL for labeling. When full cytopathic effects (CPEs) 
were observed (approximately $24 \mathrm{~h}$ post-infection), the virus in the cell supernatant after 3 freeze-thaw cycles were collected for further purification. Briefly, cell supernatants were centrifuged at 5,000 rpm for $10 \mathrm{~min}$, followed by $10,000 \mathrm{rpm}$ for $30 \mathrm{~min}$ to remove cell debris. The cleared viruses were subjected to ultra-speed centrifugation at 27,000 rpm for three hours in an SW28 rotor (Beckman, Palatine, IL USA). Sediments were dissolved in PBS buffer (150 mM, pH 7.4) and mixed with DBCO-AF647 (Jena Bioscience, Germany) or DBCOQDs for labeling at room temperature for $2 \mathrm{~h}$. Then, the labeled virus was purified by density gradient centrifugation (DGC). Briefly, the labeled virus was loaded onto a noncontinuous gradient consisting of $20 \%, 30 \%, 40 \%$, and 50\% Optiprep (Cosmo Bio, Carlsbad, CA, USA) and separated by centrifugation at $38,000 \mathrm{rpm}$ at $4{ }^{\circ} \mathrm{C}$ for four hours in an SW41 rotor (Beckman counter). Aliquots of $1 \mathrm{ml}$ each were taken from the top to the bottom of the centrifugation tube for the detection of both fluorescent signals and viral antigens.

\section{Transmission electron microscopy}

For TEM examination, carbon-coated copper grids were immersed in purified virus solution or QDs for $20 \mathrm{~min}$. Redundant liquid on the grids was removed using filter papers, and the grids were negatively stained with $1 \%$ phosphotungstate $(10 \mu \mathrm{L})$ for $10 \mathrm{~s}$. After drying at room temperature overnight, grid samples were examined under a Hitachi H7000 electron microscope.

\section{Virus titering and growth curve assay}

Viruses were titered in RD cells using the TCID50 method. Briefly, serial 10-fold diluted viruses were used to infect monolayer RD cells in 96-well plates. At $72 \mathrm{~h}$ post infection (poi), positive wells that showed CPE were counted, and virus titers were calculated using the Muench and Reed method [28]. For the growth curve assay, RD cells were infected with EV71 virus at an MOI of 0.01 . Viruses were collected at the indicated time points and then titered as described above.

\section{Immunofluorescence assay}

Cells were seeded in a $35 \mathrm{~mm}$ glass-bottom dish. After $24 \mathrm{~h}$, cells were infected with EV71. At different time points poi, cells were fixed with $4 \%$ polyformaldehyde at room temperature for $15 \mathrm{~min}$, followed by permeation for 10 min with PBS supplemented with $0.02 \%$ Triton $\mathrm{X}-100$. After that, the cells were sequentially blocked with blocking buffer (PBS containing 3\% BSA and 0.3\% normal goat serum) and incubated with EV71 specific antibody (Abcam, USA) and fluorescent secondary antibodies. Then, the cells were observed with an UltraVIEW VoX double-disc live-cell fluorescence confocal microscope
(PerkinElmer, Co). In some cases, cells were incubated with Hoechst 33,258 (Beyotime Biotech, China) to stain the nucleus before imaging. The Image pro plus software (version 6.0) was used for line profile analysis and the Fiji Image J software (imagej.nih.gov/ij/) was used for quantification of the fluorescent signals.

\section{qRT-PCR quantification of virus RNA copy number}

Viral RNA was extracted with a Viral RNA Kit (Omega Biotek, USA). To quantify viral RNA copies, a pair of VP1-specific primers and TaqMan probes were applied for one-step qRT-PCR using the SuperScript ${ }^{\mathrm{TM}}$ III OneStep RT-PCR System with Platinum ${ }^{\mathrm{TM}}$ Taq (Invitrogen, USA) on a BioRad CFX96 qPCR Machine. The sequences of primers and probes are described in [27].

\section{Drug inhibition assay}

Cells cultured in $35 \mathrm{~mm}$ glass-bottom dishes (Nest cop, China) were treated with medium containing DMSO or $50 \mathrm{mM}$ chlorpromazine. Cells were then washed and incubated with EV71-QDs at an MOI of $>50$ at $4{ }^{\circ} \mathrm{C}$ for $30 \mathrm{~min}$. Then, the cells were washed twice with ice-cold PBS, and fresh medium containing DMSO or $50 \mathrm{mM}$ chlorpromazine was added. Dishes were kept on ice until observation under an Andor spinning disk confocal microscope. During live-cell imaging, cell culture dishes were kept in a chamber that supplied $5 \% \mathrm{CO}_{2}$ at $37^{\circ} \mathrm{C}$. Trajectory and velocity analyses were performed with Fiji ImageJ using the Trackmate plugin.

\section{Results}

\section{Construction and characterization of the RD-MetRS* cell} line

An ANL-based virus labeling system was developed for the bioorthogonal labeling of viruses during infection (Scheme 1). We took advantage of the discovery that a mammalian MetRS mutant could charge methionine tRNA with ANL [25]. An expression cassette of the human MetRS mutant, termed MetRS* in this report, was inserted into the genome of RD cells to generate a cell line that could translate the ATG codon to ANL. When this cell line was used for virus production, ANL was incorporated into the viral structural protein, preparing the progeny virus for selective reaction with fluorescent molecules harboring a DBCO group through copper-free click chemistry. We first detected the ability of this cell line to incorporate ANL into cellular proteins. After integration of MetRS* into the cell genome by the PiggyBac transposon system (Fig. 1A), cells were cultured in an ANL-containing medium or normal medium as a control. Cell lysates were incubated with DBCO-AF647 dye, and the proteins were separated by sodium dodecyl sulfate-polyacrylamide gel electrophoresis. Strong AF647 


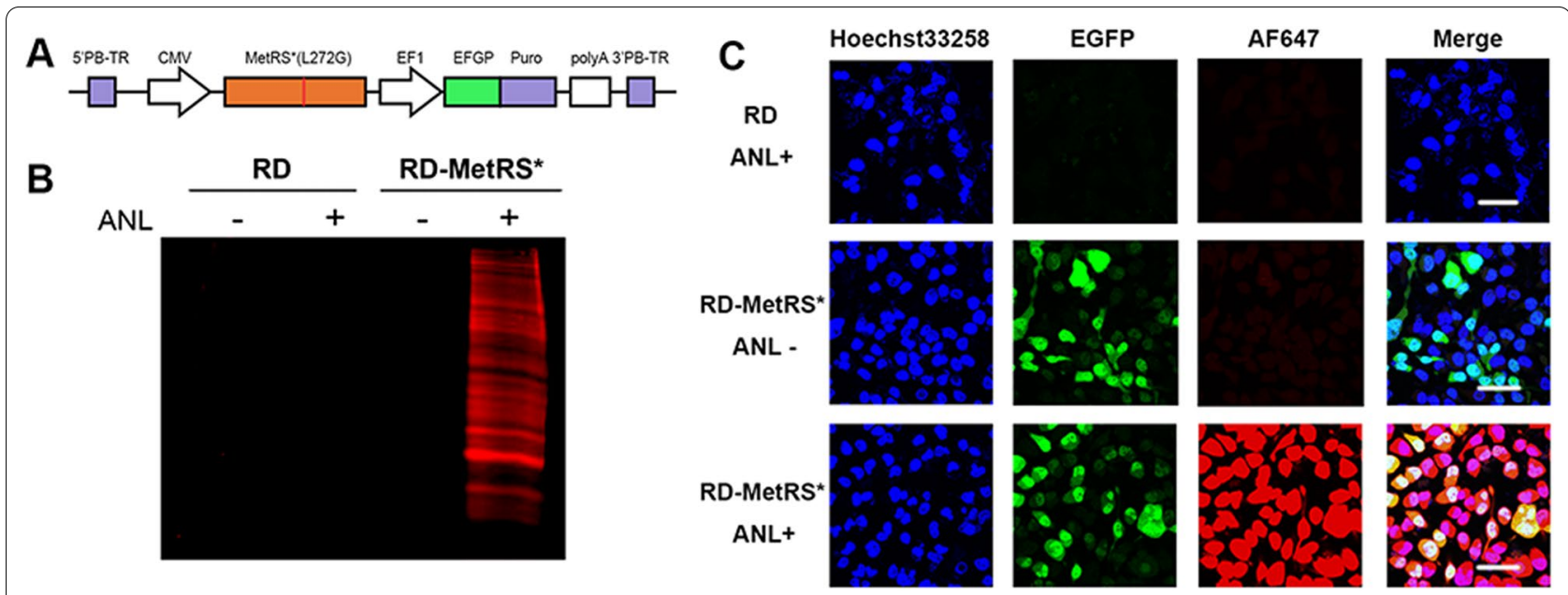

Fig. 1 Construction and characterization of the MetRS mutant-based labeling system. A Scheme of the MetRS* expression plasmid PB-MetRS*. B Sodium dodecyl sulfate-polyacrylamide gel electrophoresis for the separation of cellular proteins of RD cells (lanes 1 and 2) and RD-MetRS* cells (lanes 3 and 4) cultured with or without ANL. The gel was stained with DBCO-AF647, and fluorescent signals were detected. C Wild-type RD cells and RD-MetRS* cells cultured with or without ANL were stained with DBCO-AF647 and observed under microscopy. Scale bar: $50 \mu \mathrm{m}$

signals could be detected in RD-MetRS* cells cultured in ANL medium but not in normal medium. In contrast, the cell lysate of wild-type RD cells could not be labeled with DBCO-647, regardless of whether ANL was present (Fig. 1B). ANL incorporation was also detected by staining the cells directly with DBCO-AF647. AF647 signals could be detected in nearly all ANL medium-cultured RD-MetRS* cells but not in wild-type RD cells or RDMetRS* cells maintained without ANL (Fig. 1C). No background or nonspecific signals were detected in either the cell lysate or the labeled cells, indicating that wildtype MetRS in RD-MetRS*cannot use ANL for translation and that the MetRS* ANL pairs are highly selective. Strong AF647 signals in ANL-cultured RD-MetRS* cells also suggested the high efficiency of both the incorporation of ANL during protein synthesis and posttranslational click labeling.

\section{The MetRS* labeling system does not require methionine depletion in the medium}

Methionine in cell culture medium often competes with its functional surrogates in protein translation and reduces the efficiency of the incorporation of the UAAs as well as the labeling efficiency. When using AHA for biorthogonal labeling, cells are usually cultured in methionine-depleted medium and dialyzed FBS to enable efficient labeling $[17,19]$.

To prove that the cellular proteins can be labeled efficiently with the existence of Met through MetRS*-ANL labeling system, RD-MetRS* cells cultured in normal medium containing different concentrations of ANL were labeled with DBCO-AF647. AF647 signals gradually increased as the concentration of ANL increased from 0 to $1,000 \mu \mathrm{M}$ (Fig. 2A), suggesting a concentration dependency of the labeling efficiency. When the concentration of ANL reaches $500 \mu \mathrm{M}$, the signal of AF647 was extremely strong.

Considering the possibility that wild-type MetRS may compete with MetRS* during protein translation and translate ATG to methionine, we also determined the necessity of using a Met-free medium for virus labeling. RD-MetRS* cells were cultured in a normal or methionine-free medium containing ANL. As expected, the AF647 signals in cells cultured with $500 \mu \mathrm{M}$ ANL in the methionine-free medium were stronger than those in the normal medium group, but the cell morphology began to change when cultured with methionine-free medium for $1 \sim 2$ days (Fig. 2B). However, when the concentration of ANL was increased to $1000 \mu \mathrm{M}$, the fluorescent signals in the normal medium group were comparable to those in the methionine-free group (Fig. 2B), proving that the effect of competitive usage of methionine by cells can be simply reversed by increasing the concentration of ANL. In addition, Cell Counting Kit-8 (CCK-8) assay showed a nearly $20 \%$ decline in cell viability when using ANL as a substitute for Methionine during prolonged incubation (Fig. 2C).

Moreover, we compared the titer of EV71-GZCII strain amplified by an AHA-based system, which requires a Met-depletion medium for labeling (Met-/AHA+), and our ANL-based system (Met+/ANL+) (Fig. 2D). In the absence of UAAs, the titer of viruses amplified by an ANL-based system is about 10 times higher than that 

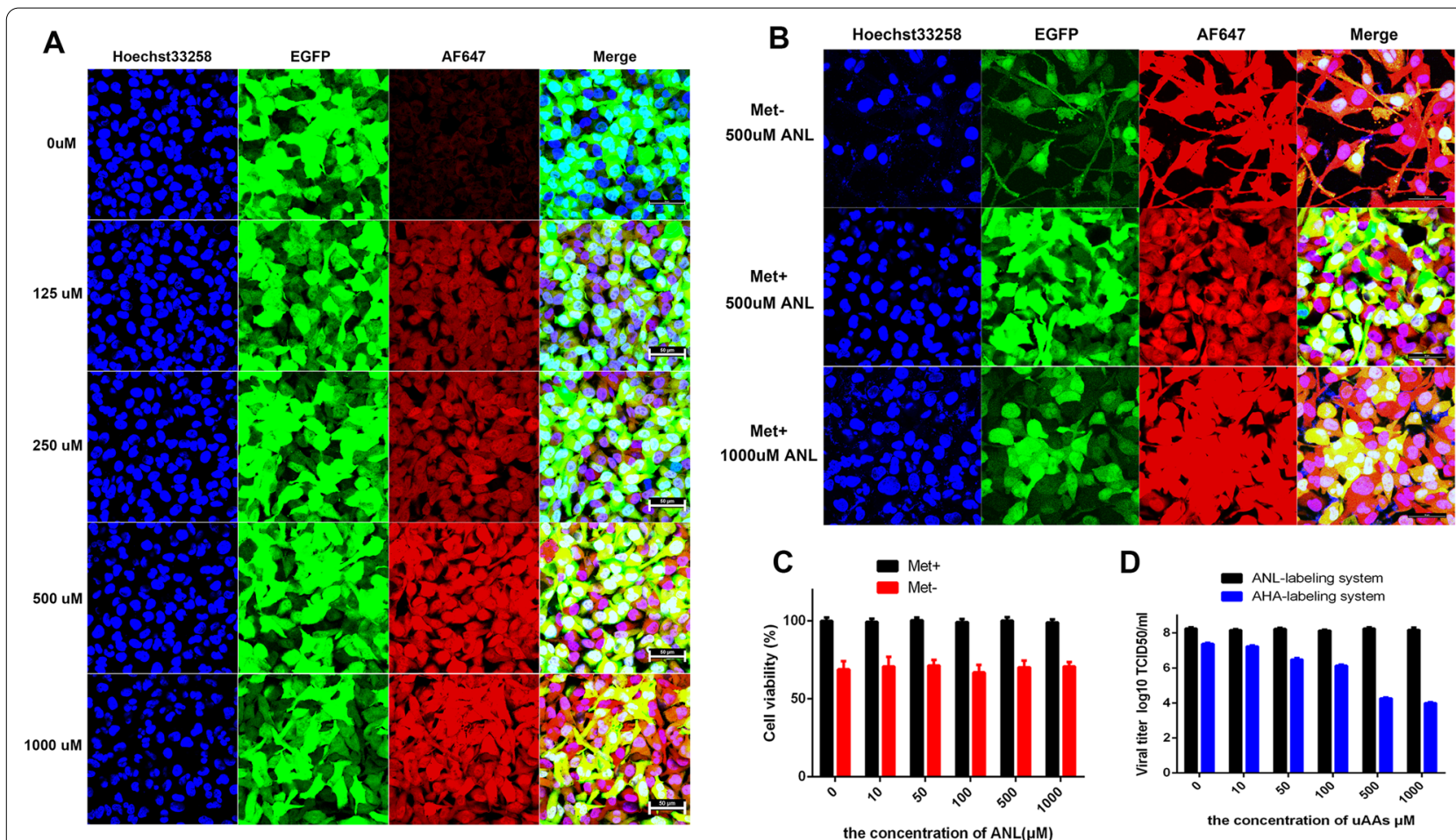

Fig. 2 Effect of methionine depletion on protein labeling and virus production. A DBCO-AF647 labeling of RD-MetRS* cells cultured in normal medium supplemented with different concentrations of ANL. Fluorescent images of RD-MetRS* cells cultured in normal or Met- medium are shown in B. Scale bar: $50 \mu \mathrm{m}$. C The viability of RD-MetRS* cells cultured in normal (Met+) or methionine-free medium (Met-) supplemented with ANL at different concentrations was detected by CCK-8 assay, and the titers of viruses produced by AHA-based system (Met-AHA+) and ANL-based system $(\mathrm{Met}+\mathrm{ANL}+)$ are shown in $\mathbf{D}$

of an AHA-based system due to no requirement of Met depletion, indicating methionine is essential for both cell growth and virus replication. When the concentration of AHA increased, the viral titer decreased significantly. Reduction of virus production by AHA in methioninefree medium was also observed during HSV labeling [21]. In contrast, no difference in cell viability (Fig. 2C) or virus yield (Fig. 2D) was observed when the ANL concentration was varied. This result is consistent with the result of the crystal violet staining assay (Additional file 1: Figure S1), indicating our ANL-based labeling system provides not only robust labeling efficiency but also high virus yield compared to other metabolic labeling strategies. And normal medium containing $1000 \mu \mathrm{M}$ ANL was used for virus labeling in the RD-MetRS* cell line.

\section{EV71 virus can be efficiently labeled with ANL and DBCO-dye}

We next determined whether EV71 could be successfully fluorescent-labeled with this system. EV71 virus was propagated in RD-MetRS* cells cultured with ANL and purified to obtain EV71-ANL for fluorescence labeling. EV71-ANL was stained by DBCO-AF647 and purified to obtain EV71-AF647 particles. To confirm whether EV71 could be specifically labeled through our system and whether EV71-AF647 particles could infect cells efficiently, HeLa cells were incubated with (a) the mixture of EV71 and DBCO-AF647, (b) the mixture of RDMetRS*(RM)-ANL cell lysates and DBCO-AF647 and (c) EV71-AF647 particles respectively and then washed to remove the dyes which cannot be uptaken by cells. Compared with the other groups, EV71-AF647 particles exhibited a different fate. As shown in Fig. 3A, cells incubated with EV71-AF647 showed strong fluorescent signals. In contrast, no signal could be observed in cells incubated with the mixture of DBCO-AF647 dye and EV71. To exclude the possibility of contamination with ANL-labeled cellular proteins, lysates of noninfected RD-MetRS* cells cultured with ANL were subjected to the same purification, labeling, and infection procedures. However, the cells in the lysate group showed no AF647 signal.

The labeling specificity was also confirmed by immunofluorescence assay (Fig. 3B). After the EV71-AF647 adsorbing on the cell surface and infecting cells for different time points, an antibody against the EV71 and 


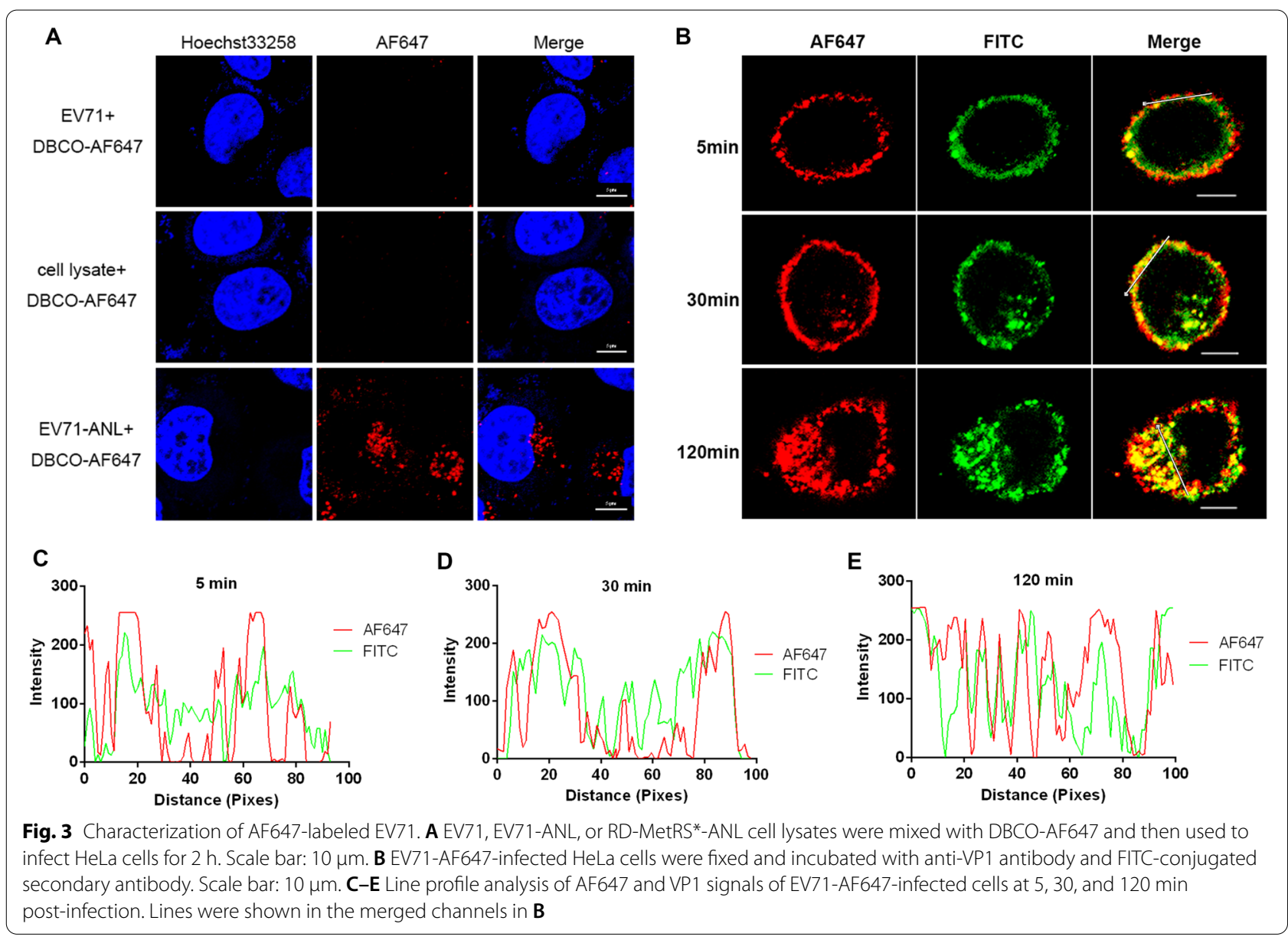

FITC-conjugated secondary antibody was added to detect viral particles. EV71-FITC signals showed high colocalization with that of DBCO-AF647, as suggested by the line profile analysis of AF647 and EV71-FITC signals on the cell surface (Fig. 3C). Moreover, both fluorescent signals moved from the cell surface toward the cell cytoplasm with increasing infection time, and the colocalization of FITC and AF647 signals at high efficiency was observed at all the indicated time points (Fig. 3CE). Thus, AF647 signals could be used to monitor virus movement in infected cells.

\section{Analysis of receptor usage by labeled EV71 virus}

EV71 virus can use multiple cellular proteins or glycans as receptors [3]. Among them, HS and SCARB2 protein were found to play important roles in the initial infection stage. To compare the usage of the two receptors by EV71, EV71-AF647 dynamics were monitored in L929 cell lines in which HS or SCARB2 was deficient due to EXT1 (essential for HS biosynthesis) or SCARB2 knockout [27]. In HS-depleted L929-EXT1KO cells, virus attachment to the cell surface was significantly reduced (Fig. 4A). Little EV71-AF647 signal was observed at either 20 or $100 \mathrm{~min}$ poi. In contrast, a strong AF647 fluorescent signal accumulated at L929 cell surface at $20 \mathrm{~min}$ poi, and some signals had shifted to the cytoplasm at $100 \mathrm{~min}$ poi (Fig. 4A). SCARB2 knockout showed only a moderate effect on EV71 attachment (Fig. 4B). The results were also supported by quantitative analysis of the AF647 signals in L929, L929-EXT1KO or L929-SCARB2KO cells (Fig. 4C-D). Interestingly, As shown in Fig. $4 \mathrm{E}$ and $\mathrm{F}$, after entering cells, the viral RNA copy number and virus titer in L929-SCARB2KO cells decreased as the infection time increased, revealing a SCARB2-dependent replication mechanism of EV71. In contrast, although HS depletion showed a strong effect on virus attachment, the viral RNA copy number or virus titer showed growth kinetics similar to those in L929-EXT1KO cells but decreased by nearly 100 -fold. Overall, the results suggested that although SCARB2 was crucial for virus infection, it was nonessential for cell attachment. It may play an essential role in EV71 uncoating or replication rather than binding and early entry stage. On 


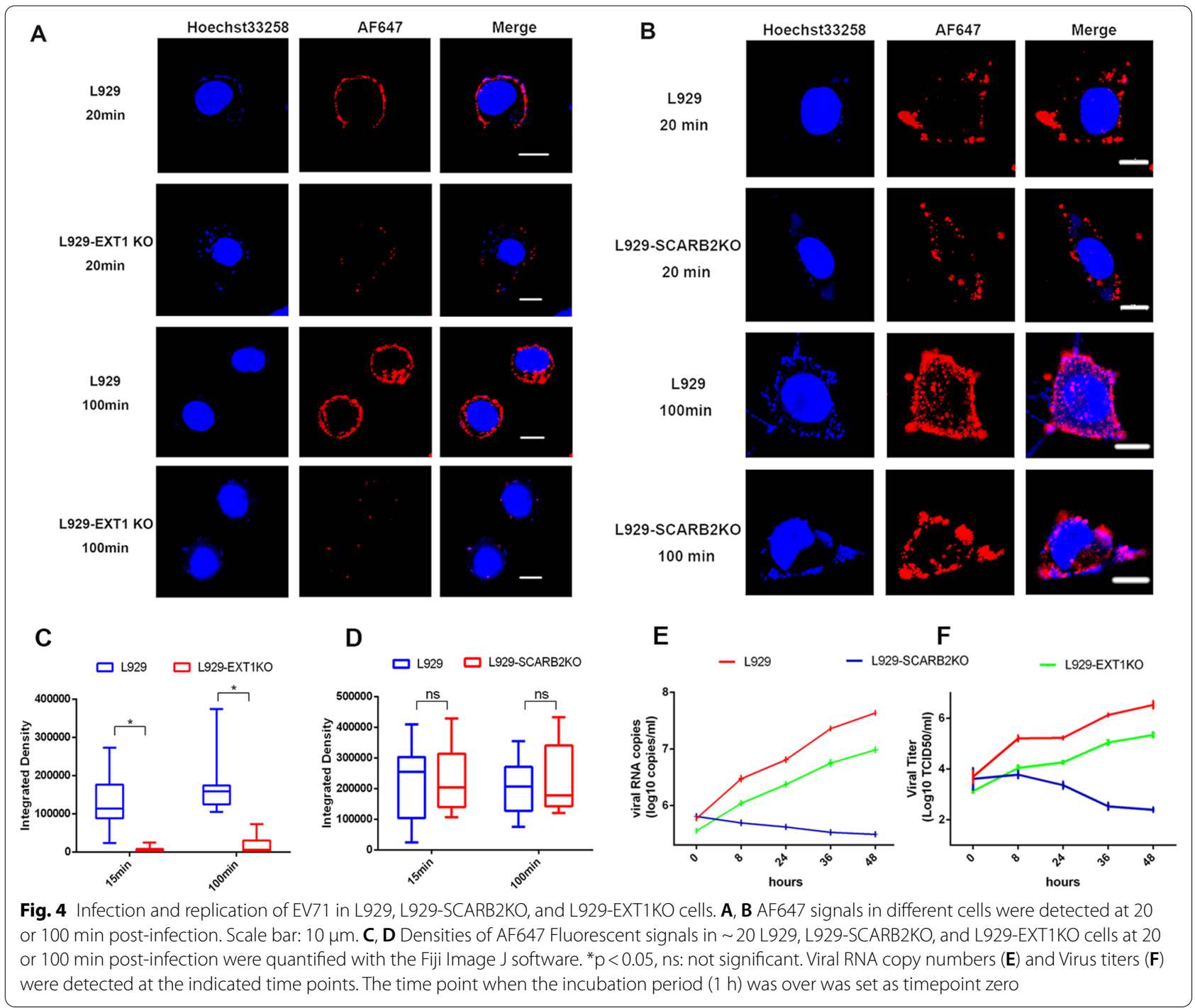

the other hand, HS plays a more important role in cell binding but cannot enable successful infection and replication without SCARB2.

\section{Characterization of QD-labeled EV71}

Chemical dyes usually undergo rapid photobleaching and are not suitable for dynamic virus tracking. QD is an alternative material that can be suitable for long-term real-time imaging owing to its remarkable brightness and photostability. The most commonly used strategy for labeling viruses with QDs is based on the reaction between streptavidin conjugated to QDs (SA-QDs) and biotin bound to the target virus. However, the size of QDs-SA can be up to $24 \mathrm{~nm}$ because of the large size of streptavidin. SA-QDs are not suitable for small-sized viruses such as EV71 which has a diameter of less than 30 $\mathrm{nm}$. Modifying QDs with small chemical groups through click chemistry ligation would not notably increase the size of QDs. Fortunately, the size of QDs can even decrease by $15 \%$ compared to QDs when conjugated with DBCO due to the hydrophobicity of DBCO [29]. Therefore, we attempted to label EV71-ANL with DBCO-QDs through click chemistry (Fig. 5). EV71-ANL was mixed with DBCO-QDs for reaction and then separated by density gradient centrifugation (DGC). DGC sample aliquots containing both QD signals under UV (Fig. 5A, B) and VP1 proteins (Fig. 5C) were observed under TEM to detect EV71-QD conjugates. Virus particles conjugated with QDs can be observed in Fig. 5F. Fluorescence spectra analysis revealed an $\sim 5 \mathrm{~nm}$ blueshift in the DBCOQDs wavelength, which may be caused by viral proteins on QDs. To determine whether the labeling procedure impairs EV71 infectivity, a growth curve assay was performed in RD cells (Fig. 5G). Compared with wild-type 


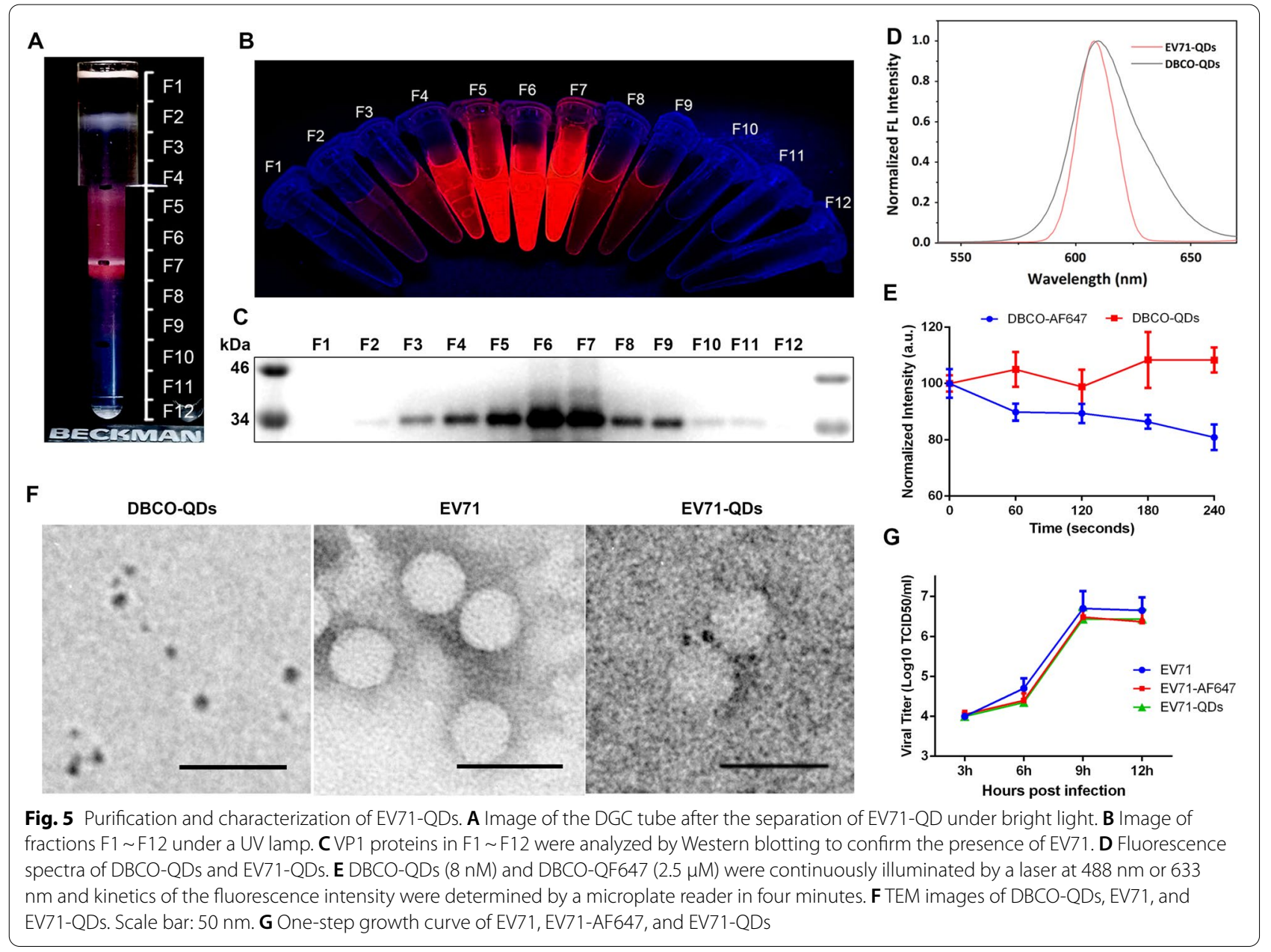

EV71 virus, both AF647- and QD-labeled viruses replicated at lower titers at different time points $(6 \mathrm{~h}, 8 \mathrm{~h}$, and $12 \mathrm{~h})$ post-infection, but the differences were not statistically significant, indicating that the labeling method did not reduce viral infectivity. Because AF647 underwent photobleaching rapidly after continuous excitation (Fig. 5E), EV71-QDs were used for dynamic visualization studies.

\section{Dynamic visualization of SCARB2-independent entry of EV71 using EV71-QDs}

Since EV71 could enter cells without SCARB2, we analyzed this entry stage through dynamic visualization using the EV71-QDs (Fig. 6). L929 or L929-SCARB2KO cells were incubated with EV71-QDs for attachment and then subjected to live cell imaging (Fig. 6). Bright QD signals were detected at the cell membrane in both cell lines at time point 0 . As time passed, the QD signals gradually moved from the periphery toward the cytoplasm. At 15 and $30 \mathrm{~min}$ post-infection,
EV71-QDs appeared mainly in the cytoplasm of L929 cells, although some fluorescent signals could still be observed at the cell surface (Fig. 6A, B). When we treated L929 cells with chlorpromazine (CPZ), an inhibitor of the clathrin-mediated endocytosis (CME) process, the entry of EV71 was blocked (Fig. 6A, C). This result is consistent with the CME entry pathway of EV71 reported previously [30]. To track the early stage of EV71 entry L929-SCARB2KO cells, we also treated the L929-SCARB2KO cells with or without CPZ before and after EV71 infection. As indicated in Fig. 6, EV71-QD signals were trapped at the cell membrane in CPZ-treated cells, but uptaken efficiently by untreated cells, regardless of whether SCARB2 was knocked out. Trajectory analysis revealed that the EV71-QDs moved toward the cell center in L929 and L929-SCARB2KO cells, which indicated virus internalization. However, in CPZ-treated cells, EV71-QDs moved in a limited area near the cell. Velocity analysis also showed a lower speed of EV71-QDs in CPZ-treated cells. Taken together, these results revealed the CME pathway of 

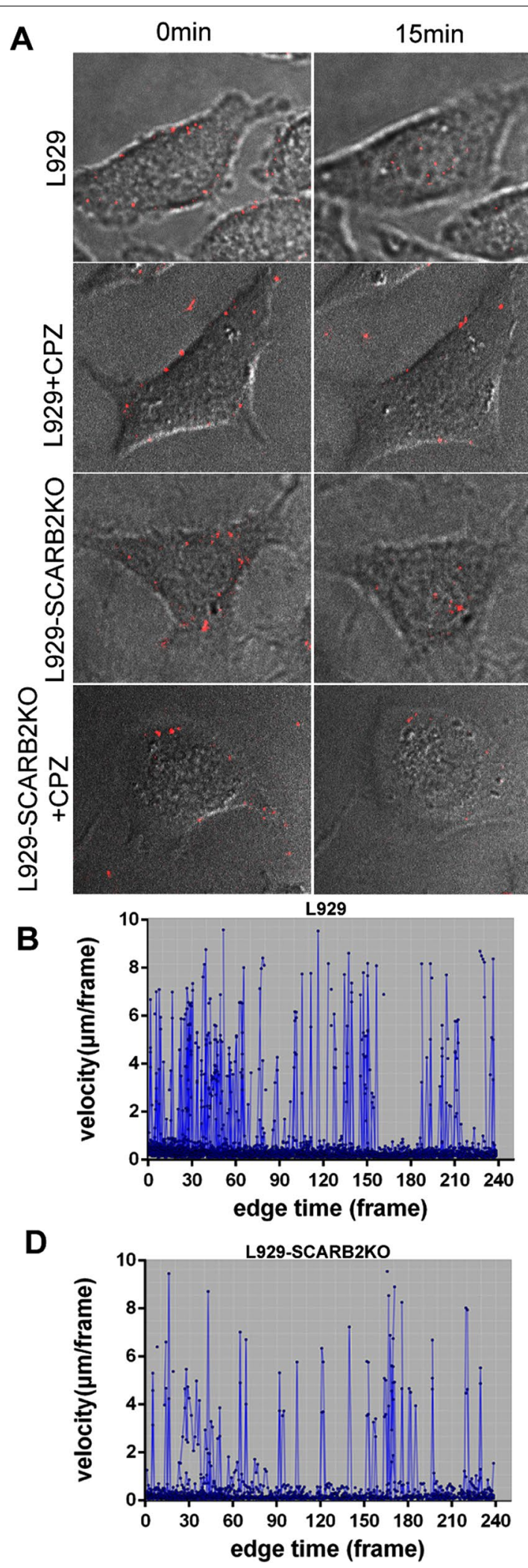

$\mathbf{E}$
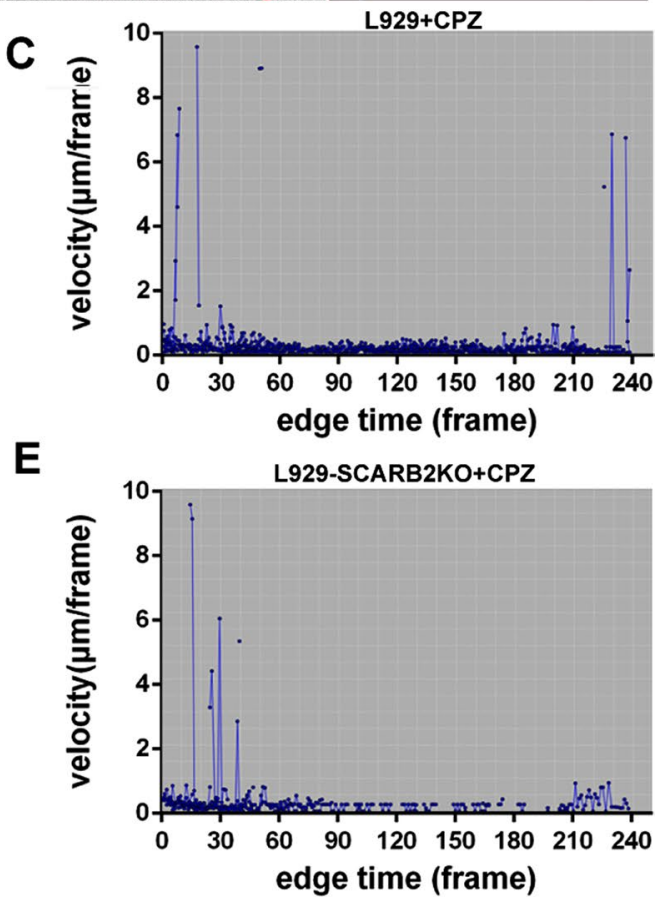

Fig. 6 Dynamics of EV71-QD movement in L929 or L929-SCARB2KO cells. L929 or L929-SCARB2KO cells infected with EV71-QDs were untreated or treated with chlorpromazine (CPZ), and the locations and trajectories (color lines) of QD signals (red dots) in cells are shown in $\mathbf{A}$. The average velocity of EV71-QDs in CPZ-treated (C, E) or untreated (B, D) cells in 240 continuous frames (in $30 \mathrm{~min}$ ) was analyzed. Scale bar: $10 \mu \mathrm{m}$ 
EV71 in SCARB2KO cells. It confirmed that as a receptor of EV71, SCARB2 is dispensable in viral attachment and early entry stage, which was not previously reported.

\section{Conclusion}

In this study, we developed a universal bioorthogonal labeling system for labeling viruses with dyes and QDs and real-time tracking of the early virus infection steps. Unlike the FP-fusion method which may prevent virus assembly, or chemical labeling which usually disrupts the charged residues essential for virus-cell interaction, our system doesn't interfere with the replication or impair the infectivity during labeling. Besides, compared with other UAA-labeling methods, our system does not require a methionine-free environment owing to the high ANL incorporation efficiency of the MetRS* cell line, thus shows no effect on cell viability and virus yield. The EV71-ANL particles can be easily labeled with DBCOprobes to track virus movements in living cells. When labeled with DBCO-QDs, the long-time real-time tracking of EV71 can be performed. In addition, this method does not require the construction of recombinant viruses for labeling through reverse genetic methods. Instead, it requires only a permissive cell line that expresses MetRS*, which can be easily constructed. Except for RDMetRS* cells, we also constructed MetRS* stably expression Vero cell line (data not shown), which can be used for propagation and labeling of many important human viruses, such as coronavirus, flavivirus, and picornavirus.

Using the labeled EV71 GZCII-P30 strain and two receptor depletion cells, we found that cellular HS is dispensable for virus replication but can significantly induce virus binding to the cell surface. On the other hand, SCARB2, another important EV71 receptor, has a limited function in cell attachment but is essential for EV71 infection and replication. Our study provides a dynamic infection process and direct evidence of the distinct functions of HS and SCARB2 in EV71 infection. Importantly, EV71 can enter SCARB2-knockout cells efficiently, probably through other receptors. And the internalization can be inhibited by chlorpromazine. We used the EV71-GZCII-P30 virus, a strong HS-binding strain, in this study. The entry mechanisms of other poor HSbinding EV71 strains may also be studied using the same method. Moreover, our findings regarding EV71 receptor utilization may also help to explain their roles in EV71 pathogenesis.

In a word, our study provides a convenient method for labeling viruses with high efficiency and robust virus yield. In principle, the method could be applied to many other viruses. Also, it takes advantage of the excellent fluorescence property of QDs and may represent a powerful tool for elucidating the molecular details of entry and intracellular transport of many kinds of viruses to study their receptors and infection mechanism.

\section{Supplementary Information}

The online version contains supplementary material available at https://doi. org/10.1186/s12951-021-01046-5.

\section{Supplementary Material}

Additional file 1: Table S1. Primers used for molecular cloning. Figure S1. The titer of EV71-GZCII produced by ANL-based system and AHAbased system detected by crystal violet staining assay.

\section{Acknowledgements}

We thank the Core Facility and Technical Support at the Wuhan Institute of Virology for technical support.

\section{Authors' contributions}

$X K$ and $Y Z$ designed the experiments, performed the experiments, curated the data, analyzed the data, and wrote the manuscript; $C L$ performed the virus purification and real-time PCR experiments; TW, DL, ZT, YL perform some of the experiments and edited the manuscript; MD and ZH provided DBCO-QDs; HW supervised the research, and edited the manuscript; $Y Z$ and $Z Z$ managed the project and edited the manuscript. All authors read and approved the final manuscript.

\section{Funding}

This work was supported by the National Natural Science Foundation of China (Grant Nos. 81871665, 81902047, 81501745, and 81702012) and the Research instrument development project of the Chinese Academy of Sciences (Grant No. YJKYYQ20190056).

\section{Availability of data and materials}

The datasets and materials used in the study are available from the corresponding author.

\section{Declarations}

Ethics approval and consent to participate

Not applicable.

\section{Consent for publication}

Not applicable.

\section{Competing interests}

The authors declare that they have no competing interests.

\section{Author details}

${ }^{1}$ CAS Key Laboratory of Special Pathogens and Biosafety, Center for Biosafety Mega-Science, Wuhan Institute of Virology, Chinese Academy of Sciences, Wuhan 430071, China. 'Department of Gastroenterology, Wuhan Children's Hospital, Tongji Medical College, Huazhong University of Science and Technology, 430015 Wuhan, China. ${ }^{3}$ The Center for Biomedical Research, Tongji Hospital, Tongji Medical College, Huazhong University of Sciences and Technology, Wuhan 430100, China. ${ }^{4}$ College of Chemistry and Molecular Sciences, Wuhan University, 430072 Wuhan, China.

Received: 3 August 2021 Accepted: 16 September 2021

Published online: 28 September 2021

\footnotetext{
References

1. Yi EJ, Shin YJ, Kim JH, Kim TG, Chang SY. Enterovirus 71 infection and vaccines. Clin Exp Vaccine Res. 2017;6:4-14.
} 
2. Marsh M, Helenius A. Virus entry: Open sesame. Cell. 2006;124:729-40.

3. Yamayoshi S, Fujii K, Koike S. Receptors for enterovirus 71. Emerg Microbes Infect. 2014;3:e53.

4. Liu Y, Rossmann MG. The cellular receptor for enterovirus 71. Protein Cell. 2014;5:655-7.

5. He QQ, Ren S, Xia ZC, Cheng ZK, Peng NF, Zhu Y. Fibronectin Facilitates Enterovirus 71 Infection by Mediating Viral Entry. J Virol. 2018:92:895.

6. Du N, Cong H, Tian H, Zhang H, Zhang W, Song L, Tien P. Cell surface vimentin is an attachment receptor for enterovirus 71. J Virol. 2014;88:5816-33.

7. Tan CW, Poh CL, Sam I-C, Chan YF. Enterovirus 71 uses cell surface heparan sulfate glycosaminoglycan as an attachment receptor. J Virol. 2013;87:611-20.

8. Liu SL, Wang ZG, Xie HY, Liu AA, Lamb DC, Pang DW. Single-virus tracking: from imaging methodologies to virological applications. Chem Rev. 2020;120:1936-79.

9. Brandenburg B, Zhuang X. Virus trafficking - learning from single-virus tracking. Nat Rev Microbiol. 2007;5:197-208.

10. Tee HK, Tan CW, Yogarajah T, Lee MHP, Chai HJ, Hanapi NA, Yusof SR, Ong KC, Lee VS, Sam IC, Chan YF. Electrostatic interactions at the five-fold axis alter heparin-binding phenotype and drive enterovirus A71 virulence in mice. PLoS Pathog. 2019;15:e1007863.

11. Tan CW, Sam I-C, Lee VS, Wong HV, Chan YF. VP1 residues around the fivefold axis of enterovirus A71 mediate heparan sulfate interaction. Virology. 2017:501:79-87.

12. Kobayashi K, Sudaka Y, Takashino A, Imura A, Fujii K, Koike S. Amino acid variation at VP1-145 of enterovirus 71 determines attachment receptor usage and neurovirulence in human scavenger Receptor B2 Transgenic Mice. J Virol. 2018;92:4.

13. Chen P, Song Z, Qi Y, Feng X, Xu N, Sun Y, Wu X, Yao X, Mao Q, Li X, et al. Molecular Determinants of Enterovirus 71 Viral Entry: Cleft Around GIn172 On Vp1 Protein Interacts With Variable Region On Scavenge Receptor B 2. J Biol Chem. 2012;287:6406-20.

14. Zhou P, Yang XL, Wang XG, Hu B, Zhang L, Zhang W, Si HR, Zhu Y, Li B, Huang $\mathrm{CL}$, et al. A pneumonia outbreak associated with a new coronavirus of probable bat origin (vol 579, pg 270, 2020). Nature. 2020;588:E6-6.

15. Pan H, Yao XJ, Chen WH, Wang FF, He HM, Liu LL, He YQ, Chen JQ, Jiang $P Z$, Zhang RL, et al. Dissecting complicated viral spreading of enterovirus 71 using in situ bioorthogonal fluorescent labeling. Biomaterials. 2018;181:199-209.

16. Dieterich DC, Hodas JJ, Gouzer G, Shadrin IY, Ngo JT, Triller A, Tirrell DA, Schuman EM. In situ visualization and dynamics of newly synthesized proteins in rat hippocampal neurons. Nat Neurosci. 2010;13:897-905.

17. Huang LL, Liu KJ, Zhang QM, Xu J, Zhao DX, Zhu HS, Xie HY. Integrating Two Efficient and Specific Bioorthogonal Ligation Reactions with Natural Metabolic Incorporation in One Cell for Virus Dual Labeling. Anal Chem. 2017:89:11620-7.

18. Lin S, Yan H, Li L, Yang M, Peng B, Chen S, Li W, Chen PR. Site-specific engineering of chemical functionalities on the surface of live hepatitis $D$ virus. Angew Chem Int Ed Engl. 2013;52:13970-4.
19. Banerjee PS, Ostapchuk P, Hearing P, Carrico IS. Unnatural Amino Acid Incorporation onto Adenoviral (Ad) Coat Proteins Facilitates Chemoselective Modification and Retargeting of Ad Type 5 Vectors. J Virol. 2011;85:7546-54

20. Bagert JD, Xie YJ, Sweredoski MJ, Qi Y, Hess S, Schuman EM, Tirrell DA. Quantitative, time-resolved proteomic analysis by combining bioorthogonal noncanonical amino acid tagging and pulsed stable isotope labeling by amino acids in cell culture. Mol Cell Proteomics. 2014;13:1352-8.

21. Serwa RA, Sekine E, Brown J, Teo SHC, Tate EW, O'Hare P. Analysis of a fully infectious bio-orthogonally modified human virus reveals novel features of virus cell entry. PLoS Pathog. 2019;15:e1007956.

22. Schmied WH, Elsasser SJ, Uttamapinant C, Chin JW. Efficient Multisite Unnatural Amino Acid Incorporation in Mammalian Cells via Optimized Pyrrolysyl tRNA Synthetase/tRNA Expression and Engineered eRF1. J Am Chem Soc. 2014;136:15577-83.

23. Joo KI, Lei Y, Lee CL, Lo J, Xie J, Hamm-Alvarez SF, Wang P. Site-specific labeling of enveloped viruses with quantum dots for single virus tracking. ACS Nano. 2008;2:1553-62.

24. Liu SL, Tian ZQ, Zhang ZL, Wu QM, Zhao HS, Ren B, Pang DW. High-efficiency dual labeling of influenza virus for single-virus imaging. Biomaterials. 2012;33:7828-33.

25. Mahdavi A, Hamblin GD, Jindal GA, Bagert JD, Dong C, Sweredoski MJ, Hess S, Schuman EM, Tirrell DA. Engineered Aminoacyl-tRNA Synthetase for Cell-Selective Analysis of Mammalian Protein Synthesis. J Am Chem Soc. 2016;138:4278-81.

26. Tom Dieck S, Muller A, Nehring A, Hinz Fl, Bartnik I, Schuman EM, Dieterich DC. Metabolic labeling with noncanonical amino acids and visualization by chemoselective fluorescent tagging. Curr Protoc Cell Biol. 2012;7:11.

27. Ke XL, Zhang Y, Liu Y, Miao YJ, Zheng CS, Luo D, Sun JH, Hu QX, Xu Y, Wang $\mathrm{HZ}$, Zheng $\mathrm{ZH}$. A single mutation in the VP1 gene of Enterovirus 71 enhances viral binding to heparan sulfate and impairs viral pathogenicity in mice. Viruses-Basel. 2020;12:33.

28. Reed H. A simple method of estimating 50\% endpoints. Am J Epidemiol. 1938:27:493-7.

29. Hao J, Huang LL, Zhang R, Wang HZ, Xie HY. A mild and reliable method to label enveloped virus with quantum dots by copper-free click chemistry. Anal Chem. 2012;84:8364-70.

30. Lin Y-W, Lin H-Y, Tsou Y-L, Chitra E, Hsiao K-N, Shao H-Y, Liu C-C, Sia C, Chong P, Chow Y-H. Human SCARB2-mediated entry and endocytosis of EV71. PLOS ONE. 2012;7:e30507.

\section{Publisher's Note}

Springer Nature remains neutral with regard to jurisdictional claims in published maps and institutional affiliations.
Ready to submit your research? Choose BMC and benefit from:

- fast, convenient online submission

- thorough peer review by experienced researchers in your field

- rapid publication on acceptance

- support for research data, including large and complex data types

- gold Open Access which fosters wider collaboration and increased citations

- maximum visibility for your research: over $100 \mathrm{M}$ website views per year

At BMC, research is always in progress.

Learn more biomedcentral.com/submissions 